\title{
Treatment Strategies for Cryptogenic Stroke Patients with Patent Foramen Ovale: What Do We Choose?
}

\author{
Yu Shen (D) ${ }^{1, *}$ \\ Qiurui $\mathrm{Nie}^{2, *}$ \\ Yibi Zhang ${ }^{3}$ \\ Qian Cao' \\ Zhuo Hou' \\ Lijun $\mathrm{Xu}{ }^{\prime}$
}

'Department of Neurology, The Second Affiliated Hospital of Nanchang University, Nanchang, Jiangxi, People's Republic of China; ${ }^{2}$ Department of Cardiovascular Medicine, People's Hospital Affiliated of Nanchang University, Jiangxi Provincial People's Hospital, Nanchang, Jiangxi, People's Republic of China; ${ }^{3}$ Department of Cardiovascular Medicine, The Second Affiliated Hospital of Nanchang University, Nanchang, Jiangxi, People's Republic of China

*These authors contributed equally to this work
Correspondence: Lijun Xu Department of Neurology, The Second Affiliated Hospital of Nanchang University, No. I, Minde Road, Nanchang, Jiangxi, People's Republic of China Email xulijun2005090।@sina.com
Importance: The treatment of cryptogenic stroke patients with patent foramen ovale to prevent recurrence of stroke, especially when patients consider drug prevention alone, has caused serious treatment dilemmas in clinical practice.

Objective: To study the safety and efficacy of different treatment strategies using a network meta-analysis of randomized controlled trials in this population with cryptogenic stroke and patent foramen ovale.

Study Selection: PUBMED, EMBASE, The Cochrane Library, WangFang, and China National Knowledge Infrastructure were searched to identify RCT comparing different treatment strategies. Eleven randomized studies were included $(n=5706)$.

Main Outcomes: The primary efficacy outcome was recurrence of ischemic stroke, including fatal and non-fatal ischemic strokes. The primary safety outcome was major hemorrhage, but closure surgery includes systemic thrombotic events, persistent atrial fibrillation, surgical deaths and other major events.

Results: In terms of efficacy and safety events, compared with antiplatelet, the OR of vitamin $\mathrm{K}$ antagonists for stroke recurrence was 0.81 (95\% CI, 0.41-1.6), the OR of surgical closure was 0.38 (95\% CI, 0.16-0.63), and the OR of NOAC was 0.79 (95\% CI, 0.27-2.3). Compared with antiplatelet, the safety event OR of vitamin $\mathrm{K}$ antagonists was $1.7(95 \% \mathrm{CI}$, $0.65-4.8$ ), the OR of surgical closure was 1.7 (95\% CI, 0.68-3.8), and the OR of NOAC was 2.2 (95\% CI, 0.67-7.6).

Conclusion: In terms of effectiveness, surgical occlusion has the best performance, while anticoagulation is the second best. Vitamin $\mathrm{K}$ antagonists and non-vitamin $\mathrm{K}$ antagonists are difficult to distinguish between the best in effectiveness. Antiplatelet drugs are considered the worst option. Regarding the safety results, it is generally believed that there are no obvious beneficial interventions, but antiplatelet drugs are considered to be relatively best, followed by surgical intervention and vitamin $\mathrm{K}$ antagonists, and non-vitamin $\mathrm{K}$ antagonists are considered to be the least safe.

Keywords: cryptogenic stroke, patent foramen ovale, PFO, treatment strategies, network meta-analysis

\section{Introduction}

Clinically, $30 \%$ of stroke patients have an unknown cause. ${ }^{1}$ Ischemic stroke of unknown cause is known as cryptogenic stroke. ${ }^{2}$ Previous studies have concluded that more than half of patients with cryptogenic stroke have a patent foramen ovale (PFO), ${ }^{3}$ and this proportion is as high as $61 \%$ in people over 55 years of age. ${ }^{4,5}$ In addition, the risk of stroke recurrence in patients with a patent foramen ovale 
increases by 3 times. ${ }^{6}$ Therefore, the prevention of stroke recurrence in such patients is particularly important. ${ }^{7}$ Although earlier clinical studies concluded that surgical closure was not superior to drug therapy alone, ${ }^{8-10}$ continuous clinical studies in recent years have concluded that surgical closure is better in reducing stroke recurrence than drug therapy alone. ${ }^{11-14}$ Subsequent surgical complications cannot be ignored, including severe thrombotic events and atrial fibrillation. ${ }^{11,13}$ Antithrombotic strategies for patients who do not choose to undergo surgical closure have always created difficulties for clinicians. Even if there was a high-quality meta-analysis that studied the difference between anticoagulation and antiplatelet therapies, it has been concluded that anticoagulation has no net benefit in preventing stroke recurrence, and it may be related to major bleeding. ${ }^{15}$ Although the studies mentioned above are important, the difference between traditional oral anticoagulant drugs and new oral anticoagulant drugs is still unclear, and whether new anticoagulant drugs are inferior to surgical closure has not been resolved. Among patients who refuse surgery, it is more difficult to select an appropriate drug to prevent stroke recurrence.

To solve this series of problems, we performed Bayesian network meta-analysis (NMA), which allows multiple treatments to be compared at the same time. This analysis included 11 randomized controlled clinical trials with a total of 5706 patients to evaluate the efficacy and safety of antiplatelet drugs, vitamin $\mathrm{K}$ antagonists, surgical closure and nonvitamin $\mathrm{K}$ antagonists (NOACs) in patients with cryptogenic stroke with a patent foramen ovale. Provide the most suitable treatment plan for such patients.

\section{Methods}

This NMA uses the systematic review and meta-analysis preferred reporting project guidelines (PRISMA) ${ }^{16}$ (eTable 1 in the Supplementary materials). A prespecified statistical analysis plan was written, and the review protocol was registered at PROSPERO (https://www.crd.york.ac.uk/PROSPERO, CRD42021272069). Data were analyzed between May 2021 and August 2021.

\section{Study Selection and Eligibility Criteria}

Two researchers conducted independent literature searches, and if there was a disagreement, it was provided to the third researcher for group discussion. The selection of the literature first involved reviewing the title and abstract, and the full-text evaluation of the studies was consistent. The PubMed, EMBASE, The Cochrane
Library (Cochrane Database of Systematic Reviews, Cochrane Central Register of Controlled Trials (CENTRAL), Cochrane Methodology Register), WangFang, China National Knowledge Infrastructure (CNKI) database was searched for relevant studies. We used keywords such as "patent foramen ovale", "treatment", and "stroke", and the time period was from the establishment of the database to May 2021. We also tried to contact the authors of valuable clinical studies where the data were not fully published. The study inclusion criteria were as follows: 1 . The study contained at least two comparison groups; 2 . The study population included patients with cryptogenic stroke with a patent foramen ovale; 3 . The study's treatment options were differentiated; 4. The outcome events included events such as the recurrence of ischemic stroke, bleeding and related surgical complications; 5 . The study follow-up period was greater than 1.5 years; and 6 . The study was a randomized controlled trial. The excluded literature included missing data, duplicate data, unclear clinical treatment plan grouping, and other nonrandomized controlled clinical trials. To ensure that the included literature was complete, we also conducted research on previous related meta-analyses.

\section{Data Collection Process and Outcome Measures}

Two researchers independently extracted literature data. The extracted content included basic information, such as the first author, year of publication, country and research design of the literature, as well as the baseline characteristics of the research population, intervention measures, and research results. The data are presented in the included attachments. The validity of the study results was defined as the recurrence of ischemic stroke, including fatal and nonfatal ischemic strokes, but it needs to be emphasized that transient ischemic attack was not included in the effectiveness outcome, which is included in this study. Most RCTs did not provide data. The safety analysis included major hemorrhage, but for closure surgery, included systemic thrombosis, persistent atrial fibrillation, surgical death and other major events. Transient arrhythmia after surgery and correctable minor bleeding were not included in the safety events. The safety analysis hybridized all malignant events. Due to the inconsistent safety of surgical events and drug events, major bleeding during surgery was relatively rare. However, surgical complications were relatively common, so the safety outcomes 
could be studied separately. Finally, we will perform a subgroup analysis of major bleeding and atrial fibrillation in the sensitivity analysis. When there was a disagreement, the disagreement was resolved through group discussion. If the included literature was not subdivided due to the intervention measures, we tried to contact the author to obtain the relevant data but did not obtain a response, so we had to exclude it from this analysis.

\section{Risk of Bias Within Individual Studies}

After that the extraction of data, two investigators independently evaluated the risk of bias for the included studies, and the risk of bias was classified as low, medium, or high. The evaluation method used was the Cochrane Collaboration tool, and Review Manager (RevMan) Version 5.3 software (Copenhagen: The Nordic Cochrane Centre, The Cochrane Collaboration, 2014) was used for this work. The risk of bias and the publication bias graph is included in the supplementary materials (eFigure 1 and eAppendix2 in the Supplement).

\section{Statistical Analysis}

We fit a Bayesian random effects model to compare multiple options at the same time. We extracted the sample size and total number of events for effectiveness and safety in each treatment group from the RCTs that were finally included in the study. The model combines the evidence of direct and indirect comparisons of related programs by considering the correlation between multiarm trials. ${ }^{17,18}$ Finally, the pros and cons of each treatment plan were drawn. To explain the heterogeneity of effects between the trials, we allowed random effects on NMA and measured the degree of heterogeneity. We used the Markov chain Monte Carlo algorithm to estimate the odds ratio (OR) of the effect of the scheme and the associated 95\% confidence interval. Through the size of each treatment group and comparison sample, each node represents a certain intervention, the size of each node represents the size of the sample, the thickness of the line represents the number of studies, and the network evidence map is drawn. Through related programs, the convergence of the Markov chain Monte Carlo chain of all model parameters was checked and the trajectory diagram was obtained. This work used the settings of the software language package, including the noninformation prior distribution of the parachain, ${ }^{19}$ and every A chain consisted of 50,000 samples after a 10,000-sample burn-in. To show that direct comparison and indirect comparison can achieve consistency in the same model, we carried out inconsistency heterogeneity tests and model comparisons and obtained the forest map and heterogeneity test results of the direct comparison and indirect comparison of the corresponding research. To evaluate and rank programs, we derived possible ranking probabilities (the best and the lowest interventions in effectiveness and safety) and a cumulative ranking table (SUCRA). Finally, to conclude that a certain intervention measured the best performance in clinical selection, we drew a two-dimensional graph so that the effectiveness and safety are clear in the graph. To prove the reliability of the outcome, we carried out a sensitivity analysis and established a new model after excluding surgical measures. Most of the data analysis and mapping were performed using R software (version 3.6.3), while the evidence contribution diagram and publication bias risk funnel diagram were generated using Stata 14.0 software. A two-sided test was used, and $\mathrm{P}<0.05$ indicated that the difference was statistically significant. Figures that do not appear in the text can be found in the attachments.

\section{Results}

\section{Search Results}

A total of 9226 articles appeared in each database search, of which 9189 articles were excluded by reviewing the titles and abstracts. A total of 137 of these studies were evaluated in full text, 13 of which met the inclusion criteria; 12 were English studies, and 1 was a Chinese study. However, two of these studies did not carry out detailed intervention measures. Although their population characteristics were consistent with this study (Figure 1), they did not provide the number of antiplatelet and anticoagulation outcome events, so we were required to exclude them from this study. Therefore, our main analysis included 11 RCTs. ${ }^{6,9-14,21,22,29-31}$ The population of the two literature studies was the same population, 9,14 but the follow-up time and the number of events that occurred at the end of the studies were different, so we consider them as two studies. Most of the studies were published in the New England Journal, and the research quality is high.

\section{Study and Patient Characteristics}

A total of 11 RCTs were included in this study. Table 1 shows the design plan, population characteristics, intervention measures and other basic characteristics of each study. The RCTs were mainly conducted in Europe and North America, and most of them were published after 2012. 
9226 studies screened

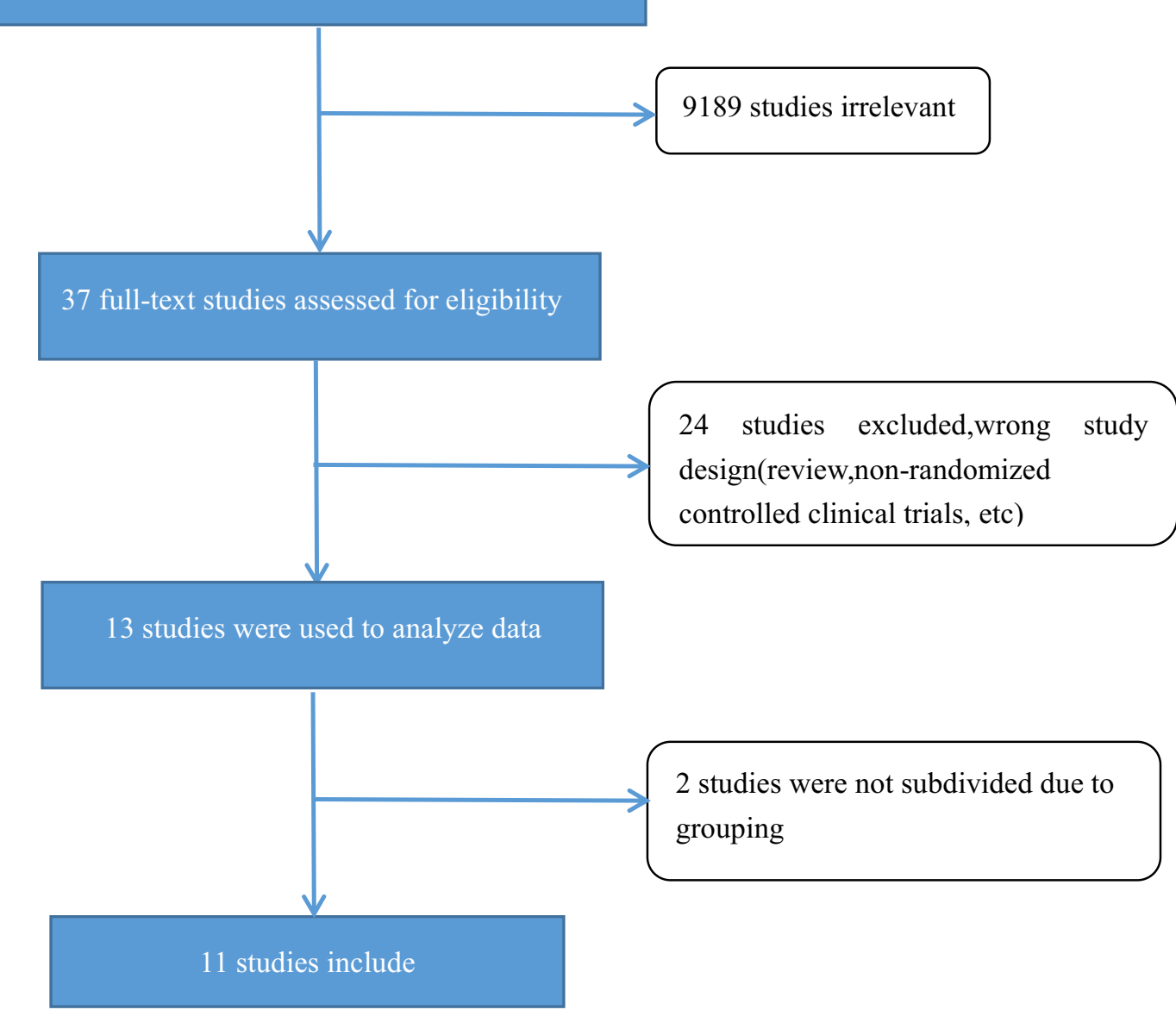

Figure I Flowchart of literature review.

This study included all 11 studies, including 5706 patients, and the sample size ranged from 44 to 980 . The safety analysis included 8 studies, including 2706 patients, and the sample size ranged from 44 to 680 . The study population was mainly 40-60 years old and was mostly white.

\section{Structure of NMA}

The main analysis of the network evidence graph is displayed by separating the effectiveness and safety (Figures 2 and 3). We compared four treatment options. The four options were antiplatelet drugs, vitamin $\mathrm{K}$ antagonists, surgical closure and nonvitamin $\mathrm{K}$ antagonists (NOACs). Because only one study subdivided single antiplatelet drugs and dual antiplatelet drugs, the use of antiplatelet drugs was considered to be a regimen. Dabigatran (a dose of $150 \mathrm{mg} / \mathrm{twice}$ a day and $110 \mathrm{mg} /$ twice a day) and rivaroxaban $(15 \mathrm{mg} /$ once a day) were also placed in the NOAC group. Therefore, in this network evidence graph, we can only assume that monoclonal antibody platelet drugs (aspirin, clopidogrel or dipyridamole) and dual antiplatelet drugs (combination of any two antiplatelet drugs) had considerable effectiveness and safety in this analysis. Dabigatran and rivaroxaban also had considerable effectiveness and safety in this analysis. We set the antiplatelet drug treatment plan as the reference plan because this plan was included the most in all the included RCTs.

\section{Network Meta-Analysis Results for Efficacy and Safety Outcomes}

We used stroke recurrence as the only indicator of effectiveness, which is also the most concerning issue for clinical stroke prevention. In general, surgical closure has been shown to be the best intervention in terms of effectiveness; only when compared with NOACs alone is there no significant difference, but it also shows obvious advantages (Figure 4, eAppendix3 Pairwise comparison of forest maps in the Supplement). We combined all major safety 


\begin{tabular}{|c|c|c|c|c|c|c|c|c|c|}
\hline$\stackrel{0}{2}$ & $\frac{\pi}{2}$ & $\frac{\pi}{z}$ & $\frac{1}{2} \frac{1}{2}$ & $\frac{s}{2}$ & & $\frac{\pi}{2}$ & $\frac{5}{5}$ & 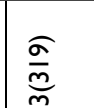 & 秀 \\
\hline & 屏 & 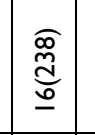 & $\frac{1}{2} \frac{\pi}{2}$ & $\frac{s}{z}$ & \begin{tabular}{|l} 
棗 \\
\end{tabular} & $\frac{s}{z}$ & $\frac{8}{2} \stackrel{\nexists}{d}$ & $\frac{\pi}{2}$ & $\frac{\pi}{2}$ \\
\hline & $\underline{\underline{\underline{\rho}}}$ & $\mid$ & $\frac{S}{z} \frac{S}{2}$ & $\frac{s}{z}$ & $\frac{\pi}{2}$ & $\overline{\tilde{\tau}}$ & 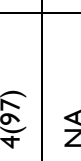 & $\frac{\pi}{2}$ & $\frac{\pi}{z}$ \\
\hline & 昰 & 愛 & $\frac{z}{z} \frac{z}{2}$ & $\frac{z}{z}$ & 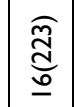 & 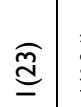 & $\frac{\widehat{d}}{\vec{b}}$ & 产 & 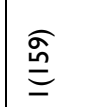 \\
\hline 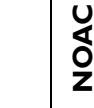 & $\frac{\pi}{z}$ & $\frac{\pi}{2}$ & \begin{tabular}{l|l}
$\Sigma$ & $\frac{S}{2}$ \\
\end{tabular} & $\frac{\mathbb{z}}{z}$ & $\frac{\pi}{2}$ & $\frac{\mathbb{z}}{z}$ & $\frac{8}{z}$ & 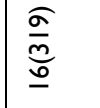 & 氮 \\
\hline & 委 & $\mid$\begin{tabular}{|c|}
$\mid$ \\
$\frac{\mathbf{w}}{2}$
\end{tabular} & 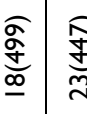 & $\frac{\widehat{d}}{\sigma}$ & 毫 & $\frac{z}{z}$ & $\frac{z}{z}$ & $\frac{\pi}{z}$ & $\mathbb{z}$ \\
\hline & $\underline{\underline{\underline{\rho}}}$ & $\mid$ & 恴 & $\overline{\overline{\underline{m}}}$ & $\frac{\pi}{2}$ & $\overline{\bar{c}}$ & 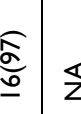 & $\frac{\pi}{2}$ & $\mid \frac{1}{z}$ \\
\hline 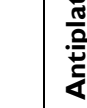 & 啜 & 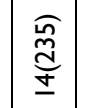 & 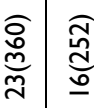 & S & \begin{tabular}{|l}
$\widehat{\widetilde{\Xi}}$ \\
$\underline{\Xi}$
\end{tabular} & $\widehat{\cong}$ & 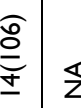 & \begin{tabular}{|l} 
产 \\
$\underline{\underline{\alpha}}$
\end{tabular} & $\mid$\begin{tabular}{|l}
$\widehat{\mathscr{d}}$ \\
$\underline{\underline{d}}$
\end{tabular} \\
\hline 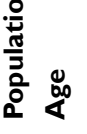 & 爰 & $\mid$\begin{tabular}{|c|}
$\overrightarrow{0}$ \\
$\vec{z}$ \\
$\vec{z}$ \\
$\vec{q}$
\end{tabular} & 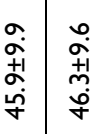 & & 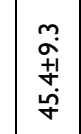 & 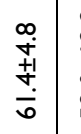 & 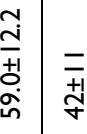 & 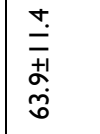 & 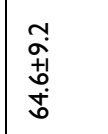 \\
\hline & 亳 & $\mid$ & 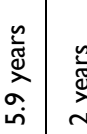 & 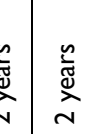 & 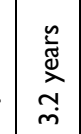 & 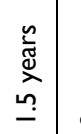 & 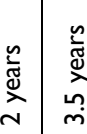 & 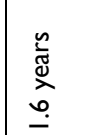 & $\frac{2}{2}$ \\
\hline 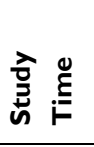 & $\begin{array}{l}\overline{\overline{\bar{\tau}}} \\
\overline{\bar{i}} \\
\overline{\bar{a}}\end{array}$ & $\mid$\begin{tabular}{|c|c|} 
\\
$\bar{a}$ \\
$\vdots$ \\
$\vdots$
\end{tabular} & 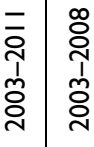 & 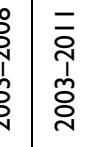 & 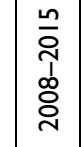 & 衰 & 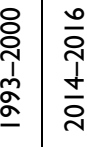 & $\begin{array}{l}\frac{\infty}{\bar{T}} \\
\frac{T}{\bar{\tau}}\end{array}$ & $\begin{array}{l}\overline{\bar{q}} \\
\frac{\bar{q}}{\bar{\alpha}}\end{array}$ \\
\hline & $\mid$ & 总 & & 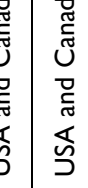 & 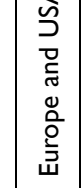 & $\underline{\underline{\underline{a}}}$ & 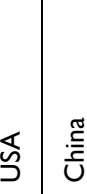 & 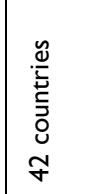 & $\begin{array}{l}\frac{\tilde{y}}{2} \\
\frac{\bar{m}}{m}\end{array}$ \\
\hline & & 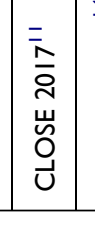 & & & & & & & \\
\hline
\end{tabular}




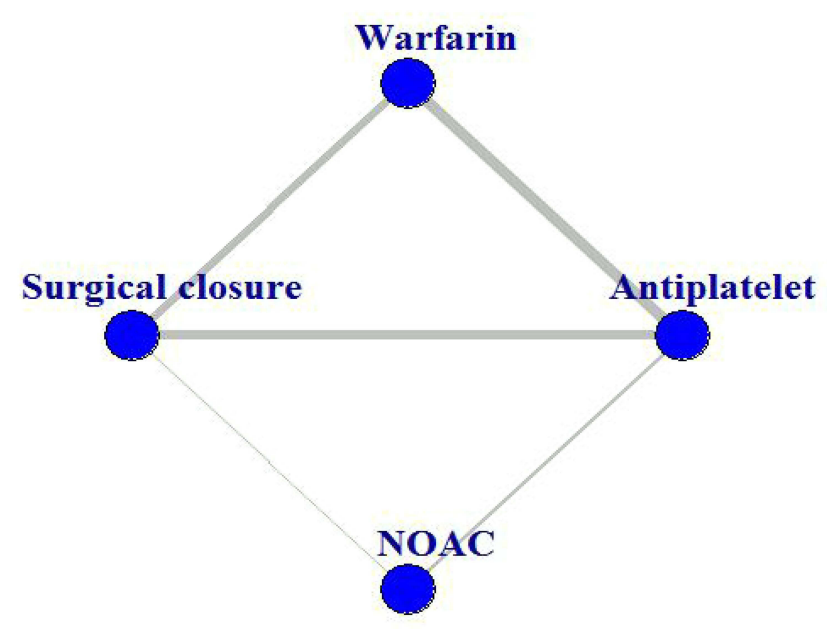

Figure 2 Network of 4 treatment strategies (Efficacy). Each node represents a certain intervention, the size of the node represents the size of the sample, and the thickness of the line represents the number of studies.

Abbreviation: NOAC, non-vitamin $\mathrm{K}$ antagonist oral anticoagulant.

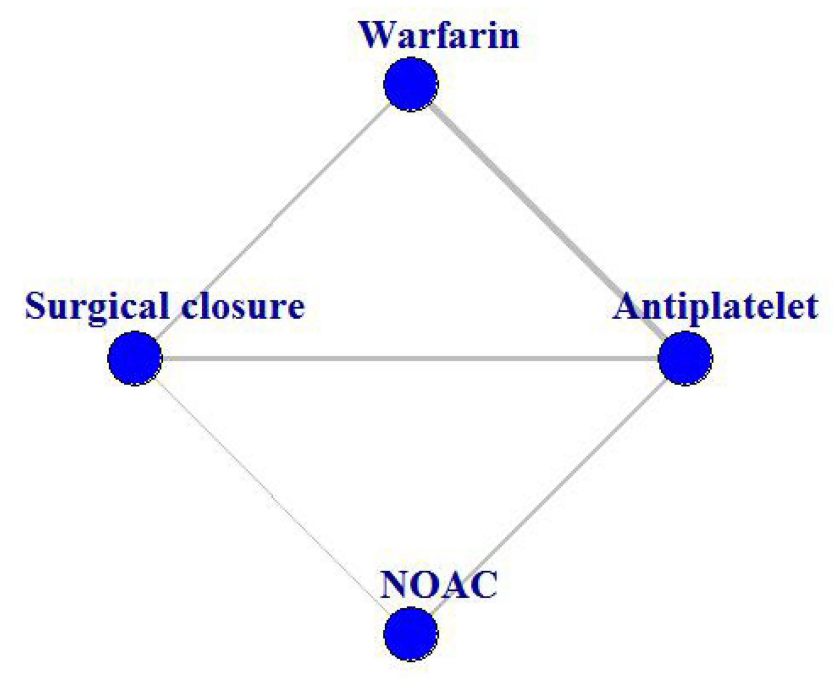

Figure 3 Network of 4 treatment strategies (Safety). Each node represents a certain intervention, the size of the node represents the size of the sample, and the thickness of the line represents the number of studies.

Abbreviation: NOAC, non-vitamin $\mathrm{K}$ antagonist oral anticoagulant.

incidents and analyzed them. In general, the direct comparison of the four interventions and the overall comparison did not show significant differences. Only the antiplatelet regimen had certain advantages, but it was not statistically significant (Figure 5, eAppendix 3 Pairwise comparison of forest maps in the Supplement).

\section{Ranking of Treatment Strategies}

In terms of ranking the results, in terms of effectiveness, surgical occlusion had the best performance (SUCRA value), while anticoagulation was the second most effective. Vitamin $\mathrm{K}$ antagonists and nonvitamin $\mathrm{K}$ antagonists were difficult to distinguish between to identify the best in effectiveness. Antiplatelet drugs were considered as the worst option (Figure 6). Summary Estimates and Rank Probabilities and Cumulative Rank Probability Plot in the Supplement (eFigures 2 and $\underline{4}$ and eTable 2 in the Supplement). Regarding the safety results, it is generally believed that there are no obvious beneficial interventions, but antiplatelet drugs are relatively considered to be relatively the safest, followed by surgical intervention and vitamin $\mathrm{K}$ antagonists, and nonvitamin $\mathrm{K}$ antagonists are considered to be the least safe (Figure 7). Summary estimates, rank probabilities and the cumulative rank probability plot are included in the Supplementary materials (eFigures 3 and $\underline{5}$ and eTable 3 in the Supplement). The treatment plan realizes the visualization of sorting on both the probability graph and the two-dimensional forest graph (Figure 8). All comparative models showed good convergence (eFigure 6 in the Supplement), and no evidence of inconsistency in this study was found (eAppendix 1 in the Supplement).

\section{Results of Sensitivity Analyses}

Analyzing the atrial fibrillation and major bleeding that are the most important regarding safety concerns, it was found that the incidence of atrial fibrillation in the surgical method was significantly higher than that of other drug groups. For major bleeding, the results for the four methods were not statistically significant, but the bleeding risk in surgery was significantly lower (eAppendix4 in the Supplement).

To verify the accuracy of the outcome and to help patients for whom surgery is not suitable choose a better intervention, we conducted additional analyses. We excluded surgical closure from the study and analyzed the three drug interventions. In terms of effectiveness, although the overall analysis did not show obvious advantages, nonvitamin $\mathrm{K}$ antagonists ranked the best, followed by vitamin $\mathrm{K}$ antagonists and antiplatelet drugs. The results for safety were the opposite of those for effectiveness. Antiplatelet drugs were the most safe, and nonvitamin $\mathrm{K}$ antagonists were the least safe. We found that the sensitivity analysis yielded similar results to the original model (eAppendix 4 in the Supplement). 


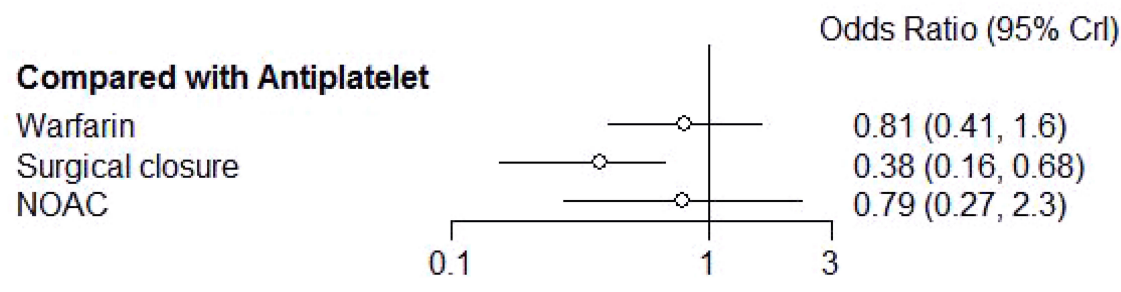

Figure 4 Forest Plots for Efficacy Outcomes. Odds ratios and $95 \%$ credible intervals (Cl) comparted with antiplatelet drugs (reference) are plotted. A total of 5706 patients were contributed to network meta-analyses for all Efficacy outcomes. The estimated between-trial effect heterogeneity and its $95 \% \mathrm{Cl}$ from $\mathrm{NMA}$ for each outcome is $0.8 \mathrm{I}$ (95\% Cl, 0.4I-I.6), 0.38 (95\% Cl, 0.16-0.63), 0.79 (95\% Cl, 0.27-2.3).

Abbreviation: NOAC, non-vitamin $\mathrm{K}$ antagonist oral anticoagulant.

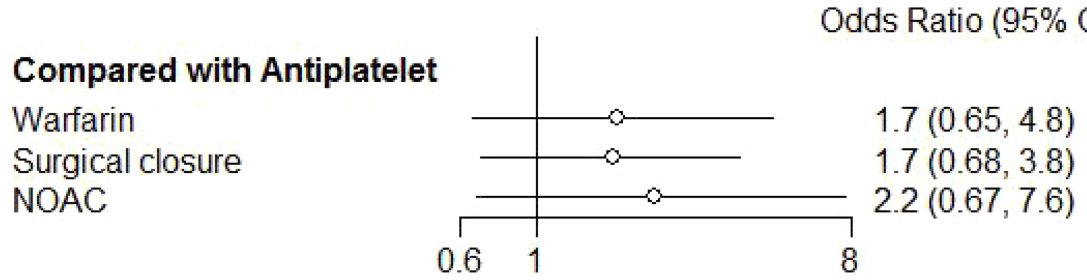

Figure 5 Forest Plots for Safety Outcomes. Odds ratios and 95\% credible intervals ( $\mathrm{Cl}$ ) comparted with antiplatelet drugs (reference) are plotted. A total of 2706 patients were contributed to network meta-analyses for all Safety outcomes. The estimated between-trial effect heterogeneity and its $95 \% \mathrm{Cl}$ from NMA for each outcome is 1.7 (95\% Cl, 0.65-4.8), I.7 (95\% Cl, 0.68-3.8), 2.2 (95\% Cl, 0.67-7.6).

Abbreviation: NOAC, non-vitamin $\mathrm{K}$ antagonist oral anticoagulant.

\section{Discussion}

For patients with cryptogenic stroke and a patent foramen ovale, the probability of a recurring stroke is extremely

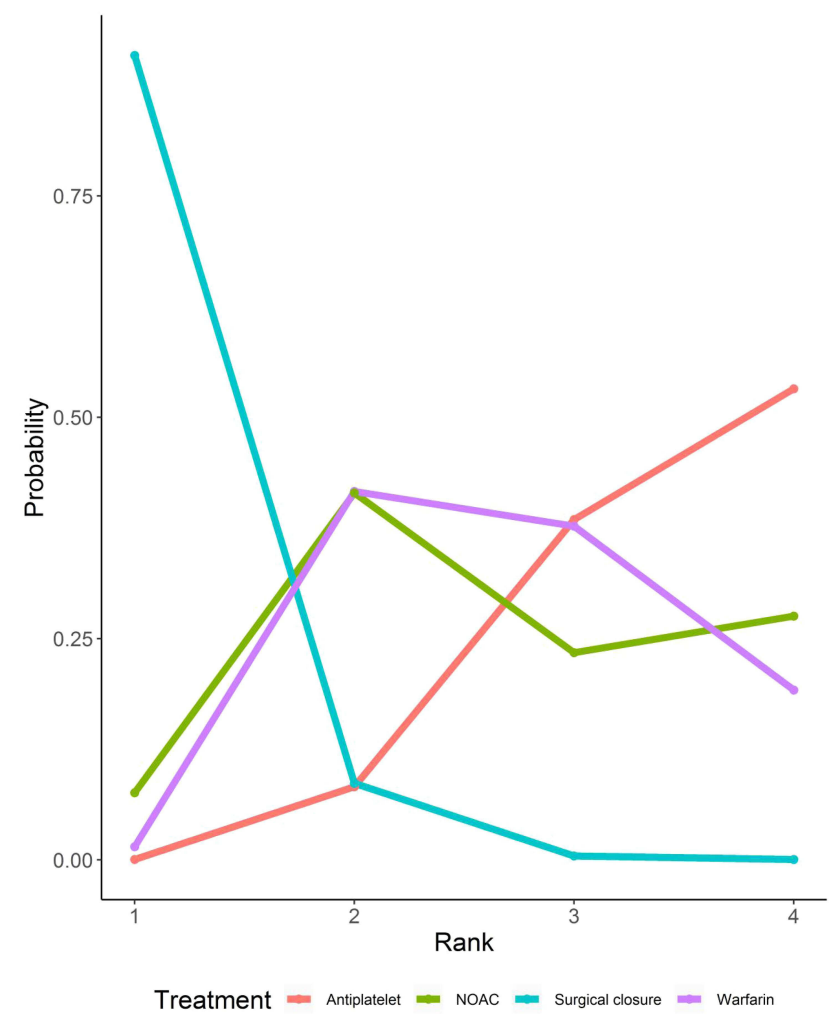

Figure 6 A ranking graph of the efficacy of each treatment plan. Abbreviation: NOAC, non-vitamin $\mathrm{K}$ antagonist oral anticoagulant. high, so active prevention is very important. To our knowledge, this is the first meta-analysis to include all four interventions. In our NMA analysis, it was concluded that surgical closure has significant effectiveness in preventing stroke recurrence. Although this method is not the best in terms of safety, the four interventions had no significant differences in safety, and surgical closure was not the least safe. Therefore, it is believed that surgical

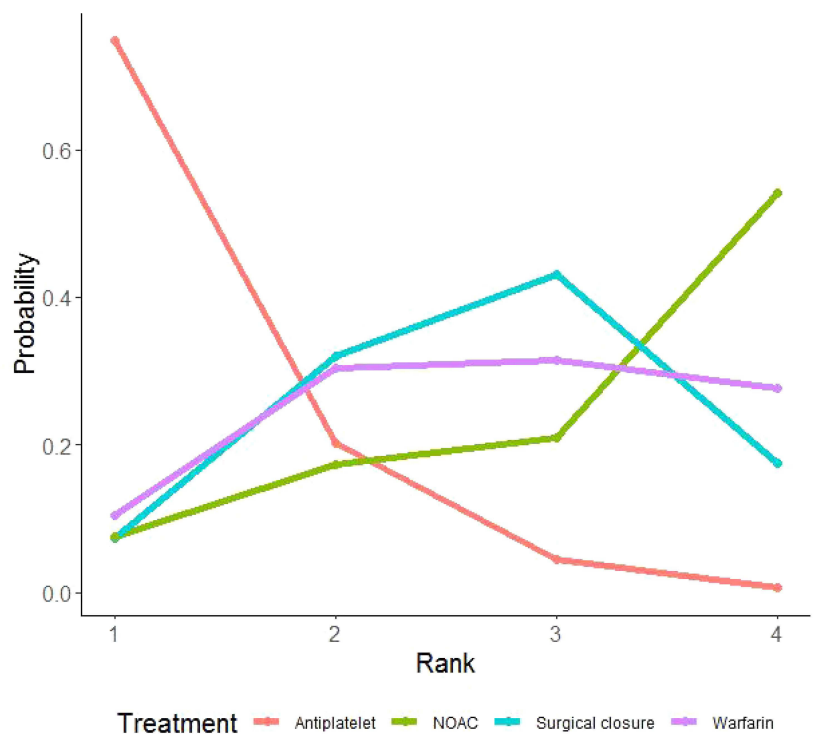

Figure $7 \mathrm{~A}$ ranking graph of the safety of each treatment plan. Abbreviation: NOAC, non-vitamin $\mathrm{K}$ antagonist oral anticoagulant. 


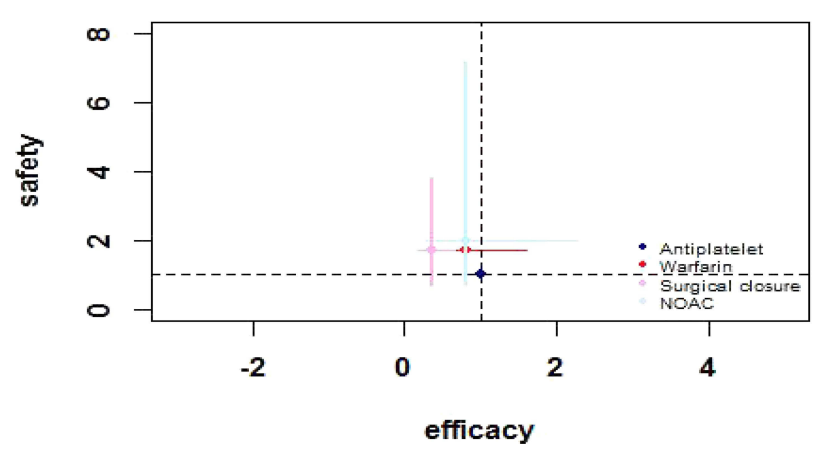

Figure 8 Odds Ratios for efficacy and safety. Odds ratios compared with antiplatelet drugs (reference) and associated $95 \%$ credible intervals are plotted: efficacy on the $x$-axis and safety on the $y$-axis.

Abbreviation: NOAC, non-vitamin $\mathrm{K}$ antagonist oral anticoagulant.

closure treatment should be regarded as the first choice of treatment, which is also consistent with the results obtained by multiple RCTs. ${ }^{11-14}$ In the meta-analysis of previous studies, ${ }^{20}$ it was found that when comparing drugs (mainly antiplatelet and vitamin $\mathrm{K}$ antagonists) with surgical methods, surgery did not significantly reduce stroke recurrence but only showed a relative trend, while the occurrence of cardiovascular and cerebrovascular events and atrial fibrillation increased. It was initially believed to be related to the immature surgical technique and the material of the occluder because the clinical studies published later showed that the effect was significant, although no significant increase in unsafe incidents was found. ${ }^{12,14}$ This supports our research results to a certain extent. Although our subgroup analysis showed that atrial fibrillation was obviously caused by surgical closure, significant results for major hemorrhage were not shown in the four strategies, and surgical closure was less likely to cause major hemorrhage. However, the comprehensive analysis of safety did not find obvious good intervention measures. These results are similar to those of previous research, ${ }^{21}$ and surgical closure is still supported as the best intervention.

However, there are also a considerable number of people who refused surgery because they were afraid of complications or economic factors, so the selection of drugs for this group of people has always been a problem. The subgroup analysis of this study found that nonvitamin $\mathrm{K}$ antagonists have the best effect in preventing stroke, but they have the opposite effect for safety. Compared with vitamin $\mathrm{K}$ antagonists, new nonvitamin $\mathrm{K}$ antagonists are promising therapeutic drugs because the concentration of these drugs in the blood is easy to detect, and they can reduce the risk of bleeding, and effectively prevent deep vein thrombosis. However, only a small number of RCTs ${ }^{22,23}$ have studied the use of these in preventive treatment, and some studies ${ }^{15}$ believe that a high anticoagulant score after an abnormal embolism score (RoPE score) supports the use of nonvitamin $\mathrm{K}$ antagonists. Because of the high RoPE score, embolism is more likely to be related to a PFO. In this study, it was concluded that nonvitamin $\mathrm{K}$ antagonists are not as safe to use as vitamin $\mathrm{K}$ antagonists. It is possible to regularly check the international normalized ratio (INR) in clinical studies to maintain the optimal level of 2-3. In actual clinical patients, this seems to be difficult. Therefore, after evaluating the RoPE score, patients with high scores should be treated with nonvitamin $\mathrm{K}$ antagonists. However, this is just speculation, and more clinical evidence is still needed in the future.

A PFO may cause ischemic stroke through the following three mechanisms: in situ thrombosis in the PFO pathway, PFO-related atrial arrhythmia, and paradoxical embolism. ${ }^{24}$ It is believed that with age, the risk of abnormal embolism further increases, but studies on surgical closure are limited. All of the RCT patients were between 40 and 50 years old, and no patients over 60 years old were included in the experiments. This is also directly related to the proneness of elderly patients to experience other thrombotic events, which increases the incidence of thrombotic events during surgery. ${ }^{25}$ Of course, we believe that the lifelong benefit of oral antithrombotic drugs for elderly patients is very relevant.

Although the advantages of our study are obvious, this study overcomes the limitations of conventional pairwise meta-analysis and visually compares the best interventions and the best drug choices for people who refuse surgery or those for whom surgery is not suitable. However, the following limitations also exist: 1. We excluded a small number of studies because we did not obtain drug grouping information, although this may not affect the final outcome; 2 . In our analysis, there was heterogeneity between the studies. Potential sources of heterogeneity include surgical methods, drug dosages, types, duration of use, and the population's sensitivity to drugs. This may affect our interpretation of the final results; and 3. Although the sample size of our study is sufficient to draw a valid final conclusion, some rare outcomes were still ignored, such as adverse reactions to the stent. This is also because the sample size for certain 
outcomes was too small. We must hybridize all events to analyze safety.

We discovered that no RCT contains a comparison between vitamin $\mathrm{K}$ antagonists and nonvitamin $\mathrm{K}$ antagonists, and there is no direct comparison between the two, which creates new difficulties for the selection of anticoagulant drugs. In the future, research in this area is needed to compare the differences between the two.

In the future, relevant meta-analyses should try to solve the shortcomings of our research, such as: 1 . Try to obtain some data that were not included in this study, including two RCTs that were not clearly grouped and were excluded after trying to contact the author; 2. Pay attention to rare adverse reactions, and conduct a more detailed subgroup analysis to reduce the number of studies heterogeneity. Of course, related clinical research should also consider the following issues: 1. Antiplatelet drug sensitivity research has shown that a large number of studies ${ }^{26-}$ ${ }^{28}$ have found that aspirin resistance and clopidogrel resistance occur in a considerable number of people. Whether antiplatelet drugs can be selectively used for people with sensitivities may be epoch-making because the safety of antiplatelets has always been beyond doubt. 2 . The individualized use of interventions for people with stroke or for the different severities of stroke. For example, severe stroke may be related to blood hypercoagulation, but anticoagulation drugs are used, and small strokes are related to abnormal small embolism, and surgical closure is selected. Of course, there are certain difficulties. In the future, we hope that personalized medical solutions based on various pieces of evidence will be available to help such patients.

\section{Acknowledgment}

Professor Lai Hengli guided our article and provided her experience in clinical work, which gave us a lot of inspiration in the process of writing the article. She is affiliated with the Department of Cardiovascular Medicine, People's Hospital Affiliated of Nanchang University, Jiangxi Provincial People's Hospital.

\section{Author Contributions}

All authors made a significant contribution to the work reported, whether that is in the conception, study design, execution, acquisition of data, analysis and interpretation, or in all these areas; took part in drafting, revising or critically reviewing the article; gave final approval of the version to be published; have agreed on the journal to which the article has been submitted; and agree to be accountable for all aspects of the work.

\section{Funding}

This research was supported by the Science and Technology Program of the Jiangxi Provincial Health Commission (202110056).

\section{Disclosure}

The authors declare no conflicts of interest for this work.

\section{References}

1. Schulze V, Lin Y, Karathanos A, et al. Patent foramen ovale closure or medical therapy for cryptogenic ischemic stroke: an updated meta-analysis of randomized controlled trials. Clin Res Cardiol. 2018;107(9):745-755. doi:10.1007/s00392-018-1224-4

2. Adams HP, Bendixen BH, Kappelle LJ, et al. Classification of subtype of acute ischemic stroke. Definitions for use in a multicenter clinical trial. TOAST. Trial of Org 10172 in Acute Stroke Treatment. Stroke. 1993;24(1):35-41. doi:10.1161/01.STR.24.1.35

3. Mojadidi MK, Zaman MO, Elgendy IY, et al. Cryptogenic stroke and patent foramen ovale. J Am Coll Cardiol. 2018;71(9):1035-1043. doi:10.1016/j.jacc.2017.12.059

4. Mas JL, Arquizan C, Lamy C, et al. Recurrent cerebrovascular events associated with patent foramen ovale, atrial septal aneurysm, or both. $N$ Engl $J$ Med. 2001;345(24):1740-1746. doi:10.1056/ NEJMoa011503

5. Handke M, Harloff A, Olschewski M, et al. Patent foramen ovale and cryptogenic stroke in older patients. $N$ Engl J Med. 2007;357 (22):2262-2268. doi:10.1056/NEJMoa071422

6. Homma S, Sacco RL, Di Tullio MR, et al.; PFO in Cryptogenic Stroke Study (PICSS) Investigators. Effect of medical treatment in stroke patients with patent foramen ovale: patent foramen ovale in Cryptogenic Stroke Study. Circulation. 2002;105(22):2625-2631. doi:10.1161/01.CIR.0000017498.88393.44

7. Amani H, Mostafavi E, Alebouyeh MR, et al. Would colloidal gold nanocarriers present an effective diagnosis or treatment for ischemic stroke? Int $J$ Nanomed. 2019;14:8013-8031. doi:10.2147/IJN. S210035

8. Meier B, Kalesan B, Mattle HP, et al. Percutaneous closure of patent foramen ovale in cryptogenic embolism. $N$ Engl J Med. 2013;368 (12):1083-1091. doi:10.1056/NEJMoa1211716

9. Carroll JD, Saver JL, Thaler DE, et al. Closure of patent foramen ovale versus medical therapy after cryptogenic stroke. $N$ Engl $J$ Med. 2013;368(12):1092-1100. doi:10.1056/NEJMoa1301440

10. Furlan AJ, Reisman M, Massaro J, et al. Closure or medical therapy for cryptogenic stroke with patent foramen ovale. $N$ Engl J Med. 2012;366(11):991-999. doi:10.1056/NEJMoa1009639

11. Mas JL, Derumeaux G, Guillon B, et al. Patent foramen ovale closure or anticoagulation vs. antiplatelets after stroke. $N$ Engl $J$ Med. 2017;377(11):1011-1021. doi:10.1056/NEJMoa1705915

12. Lee PH, Song JK, Kim JS, et al. Cryptogenic stroke and high-risk patent foramen ovale: the DEFENSE-PFO trial. $\mathrm{J}$ Am Coll Cardiol. 2018;71(20):2335-2342. doi:10.1016/j.jacc.2018.02.046

13. Søndergaard L, Kasner SE, Rhodes JF, et al. Patent foramen ovale closure or antiplatelet therapy for cryptogenic stroke [published correction appears in N Engl J Med. 2020 Mar 5;382(10):978]. N Engl $J$ Med. 2017;377(11):1033-1042. doi:10.1056/NEJMoa1707404

14. Saver JL, Carroll JD, Thaler DE, et al. Long-term outcomes of patent foramen ovale closure or medical therapy after stroke. $N$ Engl $J$ Med. 2017;377(11):1022-1032. doi:10.1056/NEJMoa1610057 
15. Romoli M, Giannandrea D, Eusebi P, et al. Aspirin or anticoagulation after cryptogenic stroke with patent foramen ovale: systematic review and meta-analysis of randomized controlled trials. Neurol Sci. 2020;41(10):2819-2824. doi:10.1007/s10072-020-04388-4

16. Hutton B, Salanti G, Caldwell DM, et al. The PRISMA extension statement for reporting of systematic reviews incorporating network meta-analyses of health care interventions: checklist and explanations. Ann Intern Med. 2015;162(11):777-784. doi:10.7326/ M14-2385

17. Dias S, Welton NJ, Sutton AJ, et al. NICE DSU technical support document 2: a generalised linear modelling framework for pairwise and network meta-analysis of randomised controlled trials. London: National Institute for Health and Care Excellence (NICE); 2014.

18. Lu G, Ades AE. Combination of direct and indirect evidence in mixed treatment comparisons. Stat Med. 2004;23(20):3105-3124. doi:10.1002/sim. 1875

19. Lopes RD, Hong H, Harskamp RE, et al. Safety and efficacy of antithrombotic strategies in patients with atrial fibrillation undergoing percutaneous coronary intervention: a network meta-analysis of randomized controlled trials. JAMA Cardiol. 2019;4(8):747-755. doi:10.1001/jamacardio.2019.1880

20. Pickett CA, Villines TC, Ferguson MA, et al. Percutaneous closure versus medical therapy alone for cryptogenic stroke patients with a patent foramen ovale: meta-analysis of randomized controlled trials. Tex Heart Inst J. 2014;41(4):357-367. doi:10.14503/THIJ-13-3879

21. Lattanzi S, Brigo F, Cagnetti C, et al. Patent foramen ovale and cryptogenic stroke or transient ischemic attack: to close or not to close? A systematic review and meta-analysis. Cerebrovasc Dis. 2018;45(5-6):193-203. doi:10.1159/000488401

22. Diener HC, Sacco RL, Easton JD, et al. Dabigatran for prevention of stroke after embolic stroke of undetermined source. $N$ Engl J Med. 2019;380(20):1906-1917. doi:10.1056/NEJMoa1813959
23. Kasner SE, Swaminathan B, Lavados P, et al. Rivaroxaban or aspirin for patent foramen ovale and embolic stroke of undetermined source: a prespecified subgroup analysis from the NAVIGATE ESUS trial [published correction appears in Lancet Neurol. 2018 Oct 23]. Lancet Neurol. 2018;17(12):1053-1060.

24. Miranda B, Fonseca AC, Ferro JM. Patent foramen ovale and stroke. J Neurol. 2018;265(8):1943-1949.

25. Homma S, Messé SR, Rundek T, et al. Patent foramen ovale. Nat Rev Dis Primers. 2016;2:15086. doi:10.1038/nrdp.2015.86

26. Xi Z, Fang F, Wang J, et al. CYP2C19 genotype and adverse cardiovascular outcomes after stent implantation in clopidogrel-treated Asian populations: a systematic review and meta-analysis. Platelets. 2019;30(2):229-240. doi:10.1080/09537104.2017.1413178

27. Patrono C, Coller B, FitzGerald GA, et al. Platelet-active drugs: the relationships among dose, effectiveness, and side effects: the Seventh ACCP Conference on Antithrombotic and Thrombolytic Therapy. Chest. 2004;126(3 Suppl):234S-264S.

28. Lu BC, Shi XJ, Liang L, et al. Platelet surface CD62p and serum vitamin D levels are associated with clopidogrel resistance in Chinese patients with ischemic stroke. J Stroke Cerebrovasc Dis. 2019;28 (5):1323-1328. doi:10.1016/j.jstrokecerebrovasdis.2019.01.031

29. Shariat A, Yaghoubi E, Farazdaghi M, et al. Comparison of medical treatments in cryptogenic stroke patients with patent foramen ovale: a randomized clinical trial. J Res Med Sci. 2013;18(2):94-98.

30. Wei Z, Wenjuan Z, Wang Y, et al. Comparison of the long-term efficacy of interventional therapy and rivaroxaban anticoagulation in patients with cryptogenic stroke with patent foramen ovale. Tianjin Med. 2019;47(8):847-850.

31. Diener HC, Chutinet A, Easton JD, et al. Dabigatran or aspirin after embolic stroke of undetermined source in patients with patent foramen ovale: results from RE-SPECT ESUS. Stroke. 2021;52 (3):1065-1068. doi:10.1161/STROKEAHA.120.031237
Neuropsychiatric Disease and Treatment

\section{Publish your work in this journal}

Neuropsychiatric Disease and Treatment is an international, peerreviewed journal of clinical therapeutics and pharmacology focusing on concise rapid reporting of clinical or pre-clinical studies on a range of neuropsychiatric and neurological disorders. This journal is indexed on PubMed Central, the 'PsycINFO' database and CAS, and is the official journal of The International Neuropsychiatric Association (INA). The manuscript management system is completely online and includes a very quick and fair peer-review system, which is all easy to use. Visit http://www.dovepress.com/testimonials.php to read real quotes from published authors. 